\title{
Diclofenac Hepatotoxicity: Case Report
}

\begin{abstract}
Héctor Bizueto-Rosas ${ }^{1, *}$, José Alberto Valdéz-Serafín ${ }^{1}$, Jesús García-Chávez ${ }^{1}$, Juan de Dios Pérez-Reyna ${ }^{1}$, Noelly Noemi Bizueto-Blancas², Déborah Gina Méndez-Alarcón², Hugo Alonso Pérez-González ${ }^{3}$, Jaime Roberto Magaña-Salcedo ${ }^{4}$, Camilo $^{2}$ Andrés Echeverry-Fernández ${ }^{4}$, Jesús Nicolás Hidalgo-Delgado ${ }^{4}$, Carlos Adrián Torrejón-Hernández ${ }^{4}$, Mariana Radilla-Flores ${ }^{4}$, Ared Mijangos-Montaño ${ }^{4}$, Joshua Ramírez-Landeros ${ }^{4}$, Carlos Daniel Gómez-Calvo ${ }^{5}$, Noemí Antonia Hernández-Pérez ${ }^{6}$, and Iván Hernández-Altamirano ${ }^{1}$
\end{abstract}

\author{
${ }^{1}$ General Surgeon, Darío Fernández Fierro General Hospital, ISSSTE, Mexico City, México \\ ${ }^{2}$ Medicine Student, Saint Luke Medicine School, Mexico City, México \\ ${ }^{3}$ Vascular Surgeon, ISSSTE, Querétaro, México \\ ${ }^{4}$ Resident, General Surgery, Darío Fernández Fierro General Hospital, ISSSTE, Mexico City, México \\ ${ }^{5}$ Vascular Surgeon, Secretaria de Salud General Hospital, Ensenada Baja California, Mexico \\ ${ }^{6}$ Family and Occupational Medicine, Instituto Mexicano del Seguro Social (IMSS) 29 Aragón, Mexico City, México
}

*Corresponding author: Bizueto-Rosas Héctor, General Surgery, Dr. Darío Fernández Fierro General Hospital, Instituto de Seguridad Social de los Trabajadores del Estado (ISSSTE), Mexico City, México, Tel: 5572288580; E-mail: dr_bizueto_h@yahoo.com

Received: 01 Apr, 2021 | Accepted: 26 Apr, 2021 | Published: 30 Apr, 2021

Citation: Bizueto-Rosas H, Valdéz-Serafín JA, García-Chávez J, Pérez-Reyna JDD, Bizueto-Blancas NN, et al. (2021) Diclofenac Hepatotoxicity: Case Report. J Surg Open Access 7(2): dx.doi.org/10.16966/2470-0991.237

Copyright: (C) 2021 Bizueto-Rosas $\mathrm{H}$, et al. This is an open-access article distributed under the terms of the Creative Commons Attribution License, which permits unrestricted use, distribution, and reproduction in any medium, provided the original author and source are credited.

\begin{abstract}
Drug-associated adverse hepatic reactions could be severe, mostly idiosyncratic, that is; they rarely occur, even if administered in the therapeutic dose and are not detected during developmental phase; any substance could be hepatotoxic, however, some drugs could have a higher potential as non-steroidal anti-inflammatory drugs and among them, diclofenac.

Objective: To present the case of a patient with the chronic intake of diclofenac and acute abdomen due to hepatic infarction.

Case Report: 58-year-old woman, admitted due to abdominal pain with two months of evolution; important background: several admissions, hypothyroidism, cigarette smoking, positive transfusions; chronic intake of diclofenac, jaundice, febrile, Murphy positive, abdominal tomography reported right pleural effusion; loculated right kidney lesion; dilated choledochus, diverticular disease; evolved with peritoneal irritation so she underwent an exploratory laparotomy that revealed primary peritonitis, reactive appendicitis, citrine fluid in cavity, whitish zones in liver segments $\mathrm{V}$ and VIII non-fluctuating; cavity wash, trans-cavity exploration of omentums and Pouchet's appendectomy was performed. Computed tomography was then reconsidered showing hypodense hepatic areas suggesting hepatic infarction. She was discharged five days postoperatively, with slight abdominal pain, afebrile, with exclusion diagnosis of hepatic infarction, secondary to chronic intake of non-steroidal anti-inflammatory drugs (diclofenac).
\end{abstract}

Conclusions: Diagnosis should be multidisciplinary, based on clinical suggestion and exclusion.

Keywords: Hepatic necrosis; Non-steroidal anti-inflammatory drugs; Diclofenac

\section{Introduction}

Drug-associated adverse hepatic reactions could be severe, mostly idiosyncratic, that is; they rarely occur, even if administered in the therapeutic dose and are not detected during developmental phase.

Any substance could be hepatotoxic; drugs with higher potential are analgesic Non-Steroidal Anti-Inflammatory Drugs (NSAIDs), commonly, diclofenac, paracetamol. Diagnosis is based on clinical suspicion, intended questionnaire and exclusion of other causes. For this reason, Hepatic Adverse Reactions (HAR) although less frequent than other adverse effects but linked to the use of these drugs, could be the cause of their removal from the marketplace [1].
It has already been described that despite idiosyncrasy; age, gender, alcohol intake, cigarette smoking, other drug's administered simultaneously, previous hepatopathy, genetic and environmental factors, are also included. Individual susceptibility depends on multiple genetic factors that modify drug's metabolization [1].

Their knowledge and early identification are important to prevent toxic reactions. Nevertheless, individual susceptibility depends on multiple genetic factors that modify the drug's metabolization [1].

\section{Diclofenac Hepatotoxicity}

Water-soluble conversion of drugs is carried out in the liver 
(detoxification), which targets it for several adverse reactions. Many drugs have been related to liver injury, however, it is not a common complication since the low incidence of idiosyncratic reactions $(1 / 100,000)$; even so, $15 \%$ of fatal hepatitis is due to drugs of difficult diagnostic because of the lack of specific markers [2]. Despite NSAIDs hepatotoxicity is low, their widespread usage and easy acquisition become a real problem. In the United States $2.2 \%$ of the patients treated per 100.000 inhabitants every year are hospitalized because of it.

The global incidence of hepatotoxicity is around 1 to 10 cases/100,000 people exposed, so, physicians should consider this group of drugs, with different chemical bases, action mechanisms, and histological patterns of damage as well as hepatotoxic potential [3].

Larrey D has reported that genetic factors are involved in DrugInduced Hepatotoxicity (DIH), in particular, changes in the HLA system for diclofenac and other drugs [4].

Additionally, other factors as a lifetime of a drug in the body, age, gender, and hormonal factors, are included.

Cytochrome P450 (CYP) is the main enzymatic system oxidizing lipophilic substrates and, together with other CYP, deactivates the molecule. When biologically active intermediates of drugs with toxic actions (phase 1 or toxifying) that release active radical species, are not blocked by type II reactions (conjugation with glucuronic or sulphuric acid, by acetylation or coupling glutathione); proteins or nucleic acids are damaged [2].

If enzymes are not enough or saturated, the drug is not cleared out correctly. Metabolites originated in phase I, could produce an immunologic reaction against the drug and the transporter protein and then produce neoantigens or cause autoimmune hepatitis. Certain host factors favor an instability between toxic metabolites production in phase I and inactivation rate (genetic predisposition, age, enzymatic induction, simultaneous diseases, idiosyncrasy, alcohol consumption, cigarette smoking, concomitant use of other drugs, previous or subjacent hepatic disease, environmental factors) [2,5].

Antibiotics, NSAIDs and anticonvulsants are among the most probable lipophilic hepatotoxic drugs [6,7].

\section{Hepatotoxicity could be classified into 2 types:}

1. Intrinsic reactions: predictable, dose-dependent, reproducible.

2. Idiosyncratic reactions (immune or metabolic): unpredictable, non-dose-dependent, affect just a few exposed patients, about $1 / 1000$ to $1 / 100,000$ [8].

Hepatic histology is the best tool to define toxicity pattern, however, in clinical practice most hepatotoxic injuries are classified with biochemical tests. Thus, according to the Council for International Organizations of Medical Sciences (CIOMS), hepatic injury exists when enzymes double the Upper Limit of Normal (ULN) [6].

\section{Injuries are then classified into:}

Hepatocellular, when there is an isolated increase of Alanine Aminotransferase (ALT) $>$ than twice of ULN or a rate between ALT/ AP (alkaline phosphatase) $>5$.

Cholestatic, isolated increase of AP $>$ than twice of ULN or a rate $<2$.

Mixed when ALT, and AP are $>$ twice of ULN or a rate $>2$, but $<5[9]$.

Mitochondrial dysfunction by inhibiting cellular respiration or any change in $\beta$-oxidation of fatty acids leads to hepatotoxicity with apoptosis, necrosis, autophagy, and cell death [9].

Main clinical-histopathological symptoms of hepatotoxicity are: an acute, chronic, fatal, cholestatic hepatitis; cholestasis (bile plugs in zone 3); vanishing bile duct syndrome; granulomatous hepatitis; macro and microvesicular steatosis, and steatohepatitis $[6,9,10]$.

Clinical manifestations of hepatotoxicity are usually non-specific: hypertransaminasemia, fever, asthenia, nausea, abdominal pain, jaundice, choluria, pruritus, even ascites, and encephalopathy $[6,11]$.

Higher incidence of hepatotoxicity due to non-selective NSAIDs has been usually reported by diclofenac and sulindac [12].

Related to diclofenac it causes acute, hepatocellular or chronic hepatitis and hepatocellular or mixed injury through a mechanism of metabolic idiosyncratic damage, leading to acute hepatitis with a long-latency period, hypertransaminasemia just in $15 \%$ of the cases, it also presents ibuprofen cross-sensitivity and is more common in women older than 60 years (as showed in our patient). Additionally, nimesulide induces a hepatocellular/cholestatic injury with the idiosyncratic immune allergic mechanism of damage, even more, in patients with hypersensitivity signs it could cause fatal hepatic failure [12]. This is a NSAID that selectively inhibits cyclooxygenase enzyme (COX-2), with low gastrointestinal toxicity; it is metabolized in the liver into 4-hydroxy nimesulide, a probably active metabolite [12].

Nitrofurantoin and omeprazole (commonly used in adults) cause hepatic injury, as well. Larrey D in 2000 reported genetic factors involved in the HLA system that predispose to diclofenac hepatotoxicity, tricyclic antidepressants, and nitrofurantoin [2].

Drug-induced hepatotoxicity is a clinical problem directly related to medical intervention, difficult to diagnose since no specific tests exist. Drug consumption in an elderly adult patients is high and usually of several drugs; so, it should be suspected with previous normal hepatic tests and when excluding other causes [11,13]. Moreover, druginduced hepatotoxicity in adults older than 50 years, means hospital admission in $40 \%$ of jaundice patients [13].

Some scales to diagnose drug hepatotixicity have been proposed, as the one from Maria VA, et al. $[13,14]$. This scale proposed a score $>17$ as definite, 14 to 17 as probable, 10 to 13 as possible, 6 to 9 indefinite and $<6$ excluded. It considers the temporal relationship between drug intake and reaction, time of drug removal before manifestations, time of drug removal before normal lab tests, exclusion of alternative causes, extrahepatic manifestations, rechallenge or accidental re冈exposure and previous report in the medical literature of toxic manifestations $[13,14]$.

Other risk factors include fatty liver; hepatic steatosis is related to hypothyroidism in $62 \%$ of the cases [15].

The case reported here was diagnosed using the recorded background in the intended questionaire as well as excluding other causes, considering the previous studies in the literature and not only with histopatological data.

\section{Clinical Case}

58-year-old women, no background of allergy, chronically degenerative diseases neither transfusions; cigarette smoking positive; abdominal hysterectomy 25 years ago, without complications.

Constant oppressive abdominal pain two months ago, treated by a particular physician with ciprofloxacin, lysine clonixinate, phenazopyridine, levofloxacin, and nimesulide. With pelvic USG a month ago, she was diagnosed with non-specified colonic pathology, 
slight diffuse steatosis, and possible right renal lipoma. Admitted in the emergency room with abdominal pain in epi, mesogastrium, and mild intensity colics; cephalea, dizziness, nausea, vomiting, and greenish evacuations.

Physical examination: Normal vital signs; pain facies, oriented, normal pupils, hydrated oral mucosa, anicteric, normal thorax, normoactive abdomen peristalsis, auscultation with pain in epigastrium, mesogastrium and right iliac fossa, Murphy negative, suspicious rebound, bilateral and positive Giordano, normal limbs.

\section{Lab tests}

Complete blood count reported hemoglobin $10.5 \mathrm{gr} / \mathrm{dl}$, hematocrit $28.9 \%$, without leukocytosis; urinalysis revealed calcium in urine and scarce bacteria (Figure 1).

Abdominal pain syndrome, chronic gastritis and urinary infection, was diagnosed.

She remains in emergency department due to possible diverticular disease, insidious evolution, plus fever $38.5^{\circ} \mathrm{C}$, abdominal distension, leukocytosis 12400, hyperbilirubinemia at the expense of the direct one, normal pancreatobiliary ultrasound; Murphy positive; splenomegaly $15 \times 4 \times 9 \mathrm{~cm}$, homogeneous echogenicity, kidneys: pelvicalyceal dilatation, right renal tumor; diagnosed as probable vesicular colic.

Vomiting appears a day later, associated with buprenorphine administration; thus, she was re-interviewed, adding a long evolution hypothyroidism background treated with levothyroxine, blood transfusion during hysterectomy; abdominal pain as constant colic, anorexia, constipation, $10 \mathrm{Kg}$ weight loss in about three months, fever $39^{\circ} \mathrm{C}$, bleeding denied.

\section{Physical examination}

Febrile, myxedematous facies; not adequately hydrated; dyspnea, tachycardia, hyperalgesia in the epigastrium and left hemiabdomen; Giordano negative. GGT $120 \mathrm{IU} / \mathrm{L}$, BD $2.28 \mathrm{mg} / \mathrm{dl}$, BI $1.16 \mathrm{mg} / \mathrm{dl}$, amylase and lipase normal, FA 328IU/L, ALT 103UI/L; leukocytes 12400, neutrophils $71 \%$, hemoglobin $8.2 \mathrm{gr} / \mathrm{dl}$, normal coagulation time. EGO: plenty of epithelial cells, amorphous urate crystals. Negative markers due to a possible renal tumor.

Torpid evolution, abdominal pain like colic, leukocyte 16.1 neutrophil 91.8\%; BT 4.41 BD 2.84, normal enzymes, antigen CA125 $62.8 \mathrm{U} / \mathrm{ml}$; CAT: right pleural effusion; right renal angiomyolipoma, dilated choledochus.

Physical examination: fever $38^{\circ} \mathrm{C}$, jaundice dye in sclera $++/+++$; dehydrated; basal bilateral hypoventilation; $4 \mathrm{~cm}$ hepatomegaly, Murphy negative, diminished peristalsis, no signs of peritoneal irritation; she was sent to internal medicine to discard hepatopathy; urology and coloproctology denied surgical problem and prescribed mesalazine and antibiotics due to Hinchey II diverticulitis. Endoscopy was required due to epigastralgy.

\section{The patient evolved with important abdominal distension,} positive rebound; lab tests

AP 318UI/L, leukocytes 15100, neutrophils $88.2 \%$, Hb 8gr/dl. She underwent an urgent exploratory laparotomy because of a possible complicated diverticular disease, finding primary peritonitis, citrine fluid in cavity, whitish zones in liver segments V and VIII nonfluctuating; reactive erythematous cecal appendix, then, abdominal wash, trans-cavity exploration of omentums through the foramen bursae omentalis and Pouchet's appendectomy was performed.

The patient was sent to internal medicine due to probable spontaneous bacterial peritonitis caused by hepatopathy. Computed tomography was re-assessed, hypodense irregular areas were noticed, suggesting hepatic infarction in segments V, VIII (Figures $2 \mathrm{~A}$ and $2 \mathrm{~B})$.

Afterwards, the patient continued with abdominal pain, without peritoneal irritation; hemodynamically stable, a febrile; oral intake acceptance; normal emunctories.
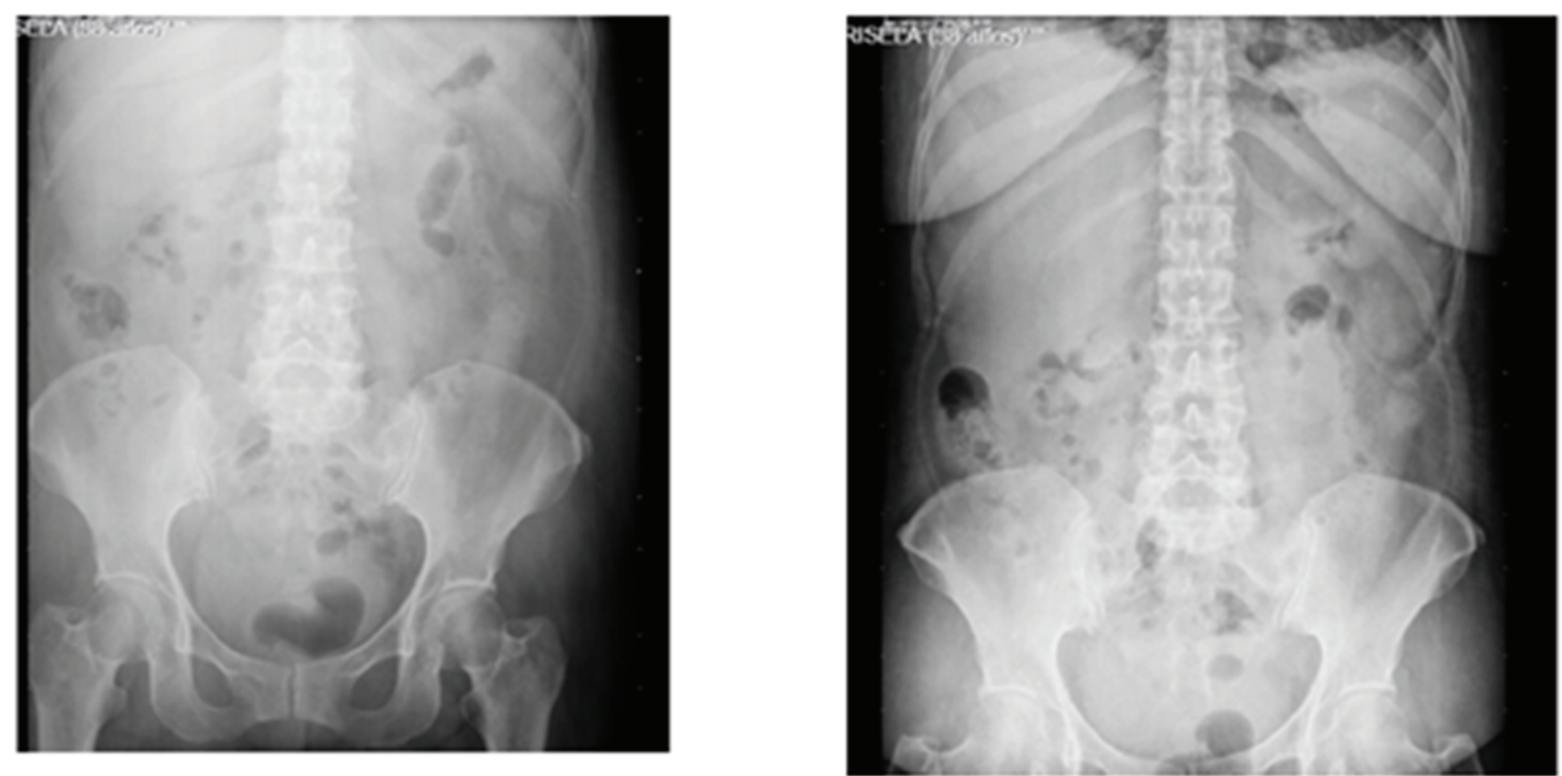

Figure 1: Simple abdomen x-ray showed abundant coprostasis; evacuating enema was performed with partial outcome. 

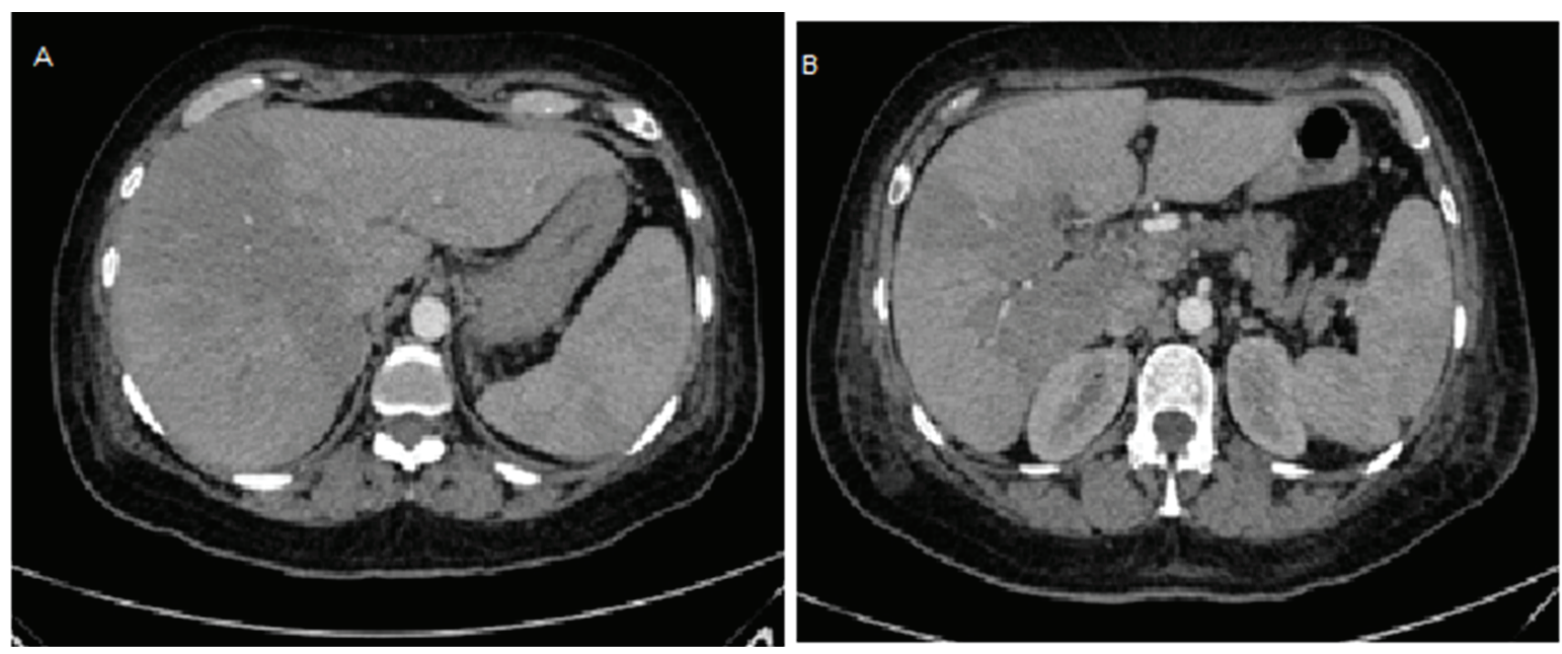

Figure 2: Simple abdomen computed tomography A) Transversal, hypodense areas, B) Infarcted areas.

BH: leukocytes 9.3, neutrophils 80\%, CGT 220, BD 2.15, BI 1.4, BT 3.55, AP 279IU/L, AST 43, ALT 17, DHL 228. EGO: urobilinogen $4 \mathrm{mg} / \mathrm{dl}$, yellow color, abundant epithelial cells, abundant amorphous urates.

\section{Peritoneal fluid cytology}

Red color, hazy appearance, $\mathrm{Hb}+++$, total cells 87,120 , leukocytes $0 \%$; erythrocytes $100 \%$; cytochemical: glucose 105 , urea nitrogen 7 , creatinine 1 , cholesterol 89 ; triglycerides 116 ; calcium 7 , potassium 3 , chlorine 108, direct bilirubin 1.0, total bilirubin 1.0.

Normal hepatobiliary ultrasound; pelvic cavity without extra data.

The patient was discharged five days postoperatively, with slight abdominal pain, afebrile, normal emunctories, and excreta.

She is remitted to gastroenterology for an exclusion diagnosis of hepatic infarction, secondary to chronic intake of NSAIDs.

\section{Discussion}

Hepatotoxic agents are classified basically into three groups: pharmacologic, pathologic, and genotoxic (DNA changes) [16].

We should also consider other risk factors for hepatic injury development as: chronic hepatic disease, simultaneous diseases, advanced age, polypharmacy, feminine gender, alcohol consumption, obesity, DM and drug addiction. Drug-induced hepatotoxicity could be due to different causes, so it should be timely identified. There are a million drugs, in the USA, 20 million people use NSAIDs either by medical or self-prescription [16].

NSAIDs usage has increased progressively in the last few years, turning them into the most used around the world [17].

NSAIDs could cause renal toxicity by inhibiting prostaglandin synthesis, even more, when the circulating volume is depleted. With this inhibition, vascular tone increases and an antinatriuretic, antireninemic, and antidiuretic effect is produced $[17,18]$.

This fortunately temporal injury could show up as an acute renal failure, interstitial nephritis, secondary hyperpotassemia, hyporeninemic hypoaldosteronism, sodium and water retention and arterial hypertension.
NSAIDs could cause hepatic toxicity in 1.1/100,000 of the patients, mainly sulindac (indole-derivative, inhibits prostaglandin production, used in pulmonary fibrosis), thus, when a transaminase increase or an albumin decrease is observed, as a default drug synthesis, they should be avoided.

Especially in elderly patients, they cause adverse neurological reactions: psychosis (indomethacin, sulindac) [17], cognitive dysfunction (loss of recent memory, inability to concentrate) and depression but they disappear weeks after drug interruption. Reactions could also be as severe as aseptic meningitis (ibuprofen) [19,20], mostly in women with Systemic Lupus Erythematosus (SLE) or even in patients without previous chronic pathologies [17].

Besides, hematological toxicity, gastric (atypical perforations of the digestive tract) and pulmonary injury have also been reported [17].

The most important is a suspected injury, discard other causes, consider self-prescription, indiscriminate use, easy access to these drugs, elder population each time, inadequate prescription, multipharmacy and physicians knowledge about this drugs with different chemical bases and its hepatotoxic potential. Biochemical parameters should be observed and immediate discontinuation should be prescribed when changes are noticed, reporting them to the corresponding authorities.

\section{NSAIDs induce hepatic injury by three mechanisms}

Host idiosyncrasy, metabolic idiosyncrasy and dose-dependent intrinsic toxicity.

Metabolic idiosyncrasy is due to hepatotoxic metabolites formation, for instance diclofenac, its active metabolite (n,5-dihydroxy diclofenac) causes direct cytotoxicity, oxidative stress, mitochondrial lesion, hepatocellular or mixed injury; hypertransaminasemia in $15 \%$ of the patients; usually its toxicity has a long-latency period (1 to 3 months) [1]. Autoimmune chronic hepatitis has also been described.

This drug should be removed from the marketplace as it has no advantages from other NSAIDs and its reactions include depression, anxiety, nightmares, psychotic reactions, aseptic meningitis, pancreatitis, intestinal diaphragms (chronic intake $>8$ weeks), constipation, intestinal pseudo-obstruction or fatal hepatitis. 
Nevertheless, other causes of hepatic injury should be discarded and exclusion diagnosis performed, since hepatic biopsy (gold standard), is not essential [21].

The liver is a target organ as it is located between the portal and systemic circulation.

Drug-induced hepatotoxicity is a growing problem, able to mimic all kinds of acute and chronic hepatic disease, besides, specific markers are still absent. A significant proportion of drug-related hepatic reactions are severe or even fatal; its incidence is unknown and just a few cases are reported to regulatory agencies [22].

Several guidelines regulate drug usage; Grupo de Trabajo de Utilización de Fármacos de la Sociedad Española de Medicina Familiar y Comunitaria (semFYC), World Health Organization prescription guideliness, CIOMS standard scale and Food Drug Administration (FDA) in America [23].

Drug Safety News Journal (July 1985), published ten guidelines for a medical prescription (Commandments for prescription), some of the most important are:

You should prescribe the least number of drugs; you should know properly the drugs prescribed; when using a new drug, get used to its pharmacology; be careful when prescribing drugs with multiple interactions (oral anticoagulants, anti-inflammatories and the ones interacting with the central nervous system); review the patients list of drugs and their possible interactions, mainly the ones expended without prescription; if a patient presents signs mismatching illness evolution, suspect about drug reactions or interactions [24].

In our patient it was never suspected, even when obvious; a retrospective analysis showed several coadjuvant causes: sex, age, and hypothyroidism (hepatic steatosis is related to hypothyroidism in $62 \%$ of the cases [15]; urosepsis treated with phenazopyridine; nimesulide and chronic intake of diclofenac. Nevertheless, it was just after surgery and intended questionnaire that the patient referred diclofenac intake for three months, 50mg twice a day for several causes, at the beginning prescribed by her general physician and later continued by her without medical approval. Moreover, emergency surgery was carried out due to patient's educational level, missing intended questionnaire and an acute abdomen without diagnostic, becoming increasingly complicated. Transoperative findings together with a non-determined etiology of hepatic lesions, previously considered as healing lesions, lead us to review the abdominal tomography thoroughly and determine, along with the radiologist, that those were hepatic infarction lesions.

Because of the severity of hepatic necrosis diagnostic by daily used drugs, primary health care is a very important factor, since the physician prescribes drugs either antibiotics or commonly analgesics without highlighting the time of intake or, on the other hand, the patient feels better and self-prescribes or use them for a long time or forever, as it happens for NSAIDs.

Primary care physicians should be fully aware and make emphasize the intake period of the drug. However, if it becomes necessary to use it for more than 8 weeks, hepatic function tests should be performed and if an increase in aminotransferase is detected, the drug should be interrupted. Mean latency period between the beginning of the treatment and the beginning of the hepatic reaction is about 12 weeks.

Prescription by tablets and not per boxes, including intake times and possible toxic reactions with therapeutic doses, is advisable.

Important to mention that no conflict of interest existed among authors since there was no risk to reveal personal information about the patient, neither ethical conflict that required informed consent.

\section{Conclusion}

Hepatic necrosis by NSAIDs could be fatal if not suspected.

If you are not properly informed about pharmacodynamics of a drug do not prescribe it.

Hepatic necrosis diagnosis could be performed by exclusion and not just by biopsy.

\section{References}

1. García-Cortés M, Andrade RJ, Lucena MI, González-Grande R, Camargo R, et al. (2006) Hepatotoxicity due to commonly-used drugs. Gastroenterol Hepatol 28: 461-472.

2. Moreno-Otero R (2002) Hepatotoxicidad por fármacos. Rev Esp Reumatol 1: s60-s71.

3. Bessone F, Tanno H (2000) Hepatotoxicity induced by non-steroidal anti-inflammatory drugs. Gastroenterol Hepatol 23: 200-205.

4. Larrey D (2000) Drug-induced liver diseases. J Hepatol 32: 77-88.

5. Lee WM (1995) Drug induced hepatotoxicity. N Engl J Med 333: 1118-1127.

6. Cano-Paniagua A, Cifuentes-Posada L, Amariles-Muñoz PJ (2017) Toxicidad hepática causada por medicamentos: revisión estructurada. Rev Colomb Gastroenterol 32: 337-348.

7. Liss G, Lewis JH (2009) Drug-induced liver injury: what was new in 2008? Expert Opin Drug Met Toxicol 5: 843-860.

8. Larrey D (2002) Epidemiology and individual susceptibility to adverse drug reactions affecting the liver. Semin Liver Dis 22: 145-155.

9. Ramachandran R, Kakar S (2009) Histological patterns in druginduced liver disease. J Clin Pathol 62: 481-492.

10. Labbe G, Pessayre D, Fromenty B (2008) Drug-induced liver injury through mitochondrial dysfunction: mechanisms and detection during preclinical safety studies. Fundam Clin Pharmacol 22: 335353.

11. Hayashi PH, Fontana RJ (2014) Clinical features, diagnosis and natural history of drug-induced liver injury. Semin Liver Dis 34: 134144.

12. Montesinos S, Hallal H, Rausell V, Conesa FJ, López A (2001) Hepatitis Aguda por nimesulida. Carta al director. Gastroenterol Hepatol 24: 219-220.

13. Contreras J, Poniachik J, Planzer M, Lazarte R, Smok G, et al. (2003) Drug induced liver disease: clinical and pathological patterns in 33 cases. Rev Med Chile 131: 1128-1134.

14. Maria VA, Victorino RM (1997) Development and validation of a clinical scale for the diagnosis of drug-induced hepatitis. Hepatology 26: 664-669.

15. Correa-Morales A, Mancera-Sánchez J, Gutiérrez-Grobe Y, UribeEsquivel M, Chávez-Tapia NC, et al. (2014) El efecto del hipotiroidismo en el hígado graso no alcohólico. Med Int Mex 30: 660-666.

16. Aguilar-Ramírez JR (2001) Hepatopatía por antiinflamatorios no esteroideos. Rev Med Hosp Gen Mex 64: S35-S37.

17. Pérez-Melón C, Armada E, Otero A, Esteban J (2000) Renal, hepatic, and neurologic toxicity caused by NSAIDs. Nefrología 20: 1-101.

18. Murray MD, Brater D (1990) Adverse effects of nonsteroidal antiinflammatory drugs on renal function. Ann Intern Med 112: 559560. 
19. Widener HL, Littman BH (1978) Ibuprofen-induced meningitis in systemic lupus erythematosus. JAMA 239: 1062-1064.

20. Lawson JM, Grady MJ (1985) Ibuprofen-induced aseptic meningitis in a previously healthy patient. West J Med 143: 386-387.

21. López-Panqueva RP (2014) Aspectos morfológicos de la enfermedad hepática inducida por drogas. Rev col gastroenterol 29: 449-460.

22. Andrade RJ, Lucena MI (2012) Hepatitis tóxica. In: Montoro MA, García Pagán JC (eds) Problemas Comunes en la Práctica ClínicaGastroenterología y Hepatología. (2 ${ }^{\text {nd }}$ Ed) Asociación Española de Gastroenterología, Madrid, España, 735-746.

23. US Food and Drug Administration (2020) Center for Drug Evaluation and Research CDER, USA.

24. Repetto-Jiménez M (1997) Interacción entre fármacos, y yatrogenia. Decálogo para el médico que receta. In: Repetto M (ed) Toxicología Fundamental ( $3^{\text {rd }}$ Ed). Madrid, España, 272-275. 